\title{
2013: a year of change for Eating and Weight Disorders - Studies on Anorexia, Bulimia, and Obesity
}

\author{
Massimo Cuzzolaro
}

Published online: 11 April 2013

(C) Springer International Publishing Switzerland 2013

Anorexia nervosa and bulimia nervosa have traditionally been regarded as entirely separate from obesity. However, the diagnostic category binge eating disorder has connected the medical area of obesity with the psychiatric field of eating disorders and, in the last 20 years, this bridge has attracted cumulative attention to the biological aspects of eating disorders, to the psycho-social and psychiatric sides of obesity and to the interface between the two fields.

A growing number of clinicians and researchers believe that the afore-mentioned polarisation is fundamentally flawed and move toward a model of shared knowledge and collaboration that considers obesity and eating disorders as two sides of the same coin. Accordingly, some authors have created wide-ranging expressions like weight-related disorders and non-homeostatic eating disorders. As a result, the process has contributed to the development of a similar multidimensional team approach to the assessment and treatment of both diseases.

Eating and Weight Disorders-Studies on Anorexia, Bulimia, and Obesity, EWD, is a quarterly peer-reviewed scientific journal whose main purpose is just to create an international forum devoted to the significant relations among the areas of eating disorders, obesity, metabolism and nutrition. This is a present and crucial topic and to enhance this core feature of the Journal, starting from the first 2013 issue, Lorenzo M Donini, Professor of Food Science and Human Nutrition, will be the Co-Editor of EWD.

The first issue of $E W D$ appeared at the end of 1996 and I wish to express my gratitude to Canzio and Alessia Fusé,

M. Cuzzolaro ( $\square)$

Formerly, University Sapienza, Roma,

Via Fedi 12, 57021 Campiglia M. (LI), Italy

e-mail: massimo.cuzzolaro@gmail.com leaders of Kurtis, the first EWD publisher, for their courage and help at the beginning of this venture. From then on, the Journal has gained increasing credit. It has an IF of 0.628 (2011) and is covered in Index Medicus/MEDLINE, PsycINFO and other well known Abstracting \& Indexing Institutes. Starting from 2010 issues, EWD has been published quarterly in the electronic version only. The months of publication are March, June, September and December.

The Journal is the official organ of the Italian Society for the Study of Eating Disorders (SIS-DCA). EWD benefits psychiatrists, psychologists, internists including endocrinologists and diabetologists, nutritional scientists, bariatric surgeons, nurses, dietitians, and others dealing with eating disorders and obesity.

Now the Journal is going through further major changes.

Starting with 2013, EWD is published by Springer Science and Business Media, the second leading international publisher of scientific, technical and medical journals and books in the world.

To increase the impact and worldwide visibility of $E W D$, electronic contents are made available through the online platform http://www.link.springer.com, which provides electronic access to more than 2,750 scientific journals, over 89,000 eBooks, more than 1,500 book series and about 250 reference works. Abstracts of published articles are freely accessible online; full-text articles are available to researchers and institutions with an electronic subscription to $E W D$ and to SIS-DCA members. Through arrangements with more than 400 consortia deals as well as a progressive development strategy in emerging markets such as China, India, Springer can further increase the readership of $E W D$.

Furthermore, to offer outstanding support to Authors and readers, Springer provides EWD with Editorial Manager (EM), one of the leading web-based manuscript 
submission systems available today. EM allows Authors to submit online their manuscripts, provides online peer review process and tracks manuscripts through the whole review process until acceptance. Moreover, EM is fully compliant with Springer's production workflows to optimize publication times: articles accepted in Editorial Manager are made available within 3 weeks upon acceptance, as Online First articles on the Springer platform http://www.link.springer.com, granting their immediate visibility and citability through their DOI number.

A journal can only be as good as its editorial team, and aiming to achieve rapid growth of this publication, I hope that each member of the Board will contribute actively to the development of $E W D$. I wish that our work will be seminal and fruitful for our readers. 